\title{
Ethnodirigid study of Medicinal plants used by the population assisted by the "Programa de Saúde da Família" (Family Health Program) in Marechal Deodoro - AL, Brazil
}

\author{
J. C. Lima Melro ${ }^{a}$ (D), S. A. Fonseca ${ }^{\text {(D) }, ~ J . ~ M . ~ S i l v a ~ J u ́ n i o r ~}{ }^{a}$ (D), S. P. B. Franco ${ }^{a}$ (D), \\ M. A. Souza ${ }^{a}$ (D) J. G. Costa ${ }^{a}$ (D), Y. F. C. Pimentel ${ }^{a}$ (D), M. R. P. Bomfim ${ }^{a}$ (D), E. M. Almeida ${ }^{a}$ (D), \\ T. J. Matos-Rocha ${ }^{a, b}$ (D), A. F. Santos ${ }^{a, c *}$ (D) \\ ${ }^{a}$ Centro Universitário Cesmac, Rua Cônego Machado, 918, Farol, CEP 57051-160, Maceió, AL, Brasil \\ 'úcleo de Ciências Biológicas, Universidade Estadual de Ciências da Saúde de Alagoas, Rua Doutor Jorge de Lima, 113, \\ Trapiche da Barra, CEP 57010-382, Maceió, AL, Brasil \\ 'Universidade Estadual de Alagoas, R. Gov. Luís Cavalcante, s/n, Alto do Cruzeiro, CEP 57312-000, Arapiraca, AL, Brasil \\ *e-mail: aldenirfeitosa@gmail.com
}

Received: September 13, 2018 - Accepted: February 15, 2019 - Distributed: May 31, 2020

\begin{abstract}
This study aimed to assess diagnosis of Unique Health System (SUS) users profile with regard to herbal medicine. This is a cross-sectional study with observational data collection conducted in the city of Marechal Deodoro, through a semi-structured questionnaire with questions related to socioeconomic data, use, and knowledge of medicinal plants and herbal medicines and satisfaction with the use of medicinal herbs $(\mathrm{MH})$ and interactions of phytotherapeutic drugs (PD). Among the topics, $86.89 \%$ of users used herbal medicines often, especially lemon balm (Melissa officinalis L.). It was also observed that the plants are generally used as a sedative $(21.74 \%)$ and as tea $(87.84 \%) .82 .32 \%$ of respondents claim to have acquired the knowledge of herbal medicine through a family member and $85.36 \%$ make use of medicinal plants without having received any information on the use of $\mathrm{MH}$ and PD. $61 \%$ of the plants described that coincided with the form of use and therapeutic properties found in the literature. With regard to satisfaction by treatment with $\mathrm{MH}$ and $\mathrm{PD}$, it was found that $56.67 \%$ are satisfied. Therefore, it is necessary to pay attention to this aspect of usage, in order to avoid possible drug interactions or adverse reactions resulting from the incorrect use.
\end{abstract}

Keywords: medicinal plants, community, phytotherapeutics, family health program.

\section{Estudo etnodirigido de Plantas medicinais utilizadas pela população atendida no "Programa de Saúde da Família”, Marechal Deodoro, AL, Brasil}

\begin{abstract}
Resumo
Este trabalho teve como objetivo conhecer perfil diagnóstico de usuários do SUS com relação à fitoterapia. Trata-se de um estudo observacional transversal, com coleta de dados realizada no Município de Marechal Deodoro, por meio de um questionário semi-estruturado com perguntas relativas a dados socioeconômicos, utilização e conhecimentos sobre plantas medicinais e fitoterápicos e satisfação com o uso de plantas medicinais (PM) e medicamentos fitoterápicos (MF). Entre os usuários abordados, 87,08\% utilizam plantas medicinais frequentemente, principalmente a erva cidreira (Melissa officinalis L.). Também foi observado que as plantas são geralmente usadas como calmante (16,05\%) e na forma de chá (68,35\%). 82,51\% dos entrevistados afirmam ter adquirido o conhecimento sobre fitoterapia através de algum familiar e 85,56\% fazem uso das PM e MF sem ter recebido algum tipo de informativo sobre o seu uso. $61 \%$ das plantas descritas pelos usuários teve seu uso e terapêutica coincidente com o citado em literatura específica. Com relação a satisfação através do tratamento com PM e MF observou-se que $56,80 \%$ estão satisfeitos. O uso de plantas medicinais por parte dos usuários é bastante frequente, principalmente pelo conhecimento acumulado ao longo de gerações. Portanto, se faz necessária maior atenção a esse aspecto de utilização, visando evitar possíveis reações adversas advindas do uso incorreto.
\end{abstract}

Palavras-chave: plantas medicinais, comunidade, fitoterapia, programa de saúde da família.

\section{Introduction}

Medicinal plants have been the main curative agents used since ancient medicine. According to the World Health Organization, about $80 \%$ of people still rely on traditional medicine, and today's demand is rising because of poverty and lack of access to modern medicine (Nolla et al., 2005).

Integrative and complementary practices (PICs) comprise a set of systems, practices and clinical use 
products, not considered as conventional medical practice. After the publication the national policy for Integrative Practices and Complementary (PNPIC), homeopathy, the medicinal plants and herbal remedies, medicine Chinese traditional/acupuncture, anthroposophical medicine and social thermalism-crenotherapy institutionalized in the system were only of health (SUS). In this sense, the Organization World Health Organization (who) defines Medicine

Traditional (MT) and alternative medicine and Complement (MAC) as practices that include medication therapies, which use herbs, animal parts or minerals, and therapies without medication, for example, acupuncture and therapy spiritual (Bragança, 1996; Eldin and Dunford, 2001).

This perception about the curative power of medicinal plants and the relationship between practices related to habitual use is often observed within traditional communities (Jacoby et al., 2002). According to estimates, people and traditional communities (indigenous and rural) occupy almost $25 \%$ of the Brazilian territory (Coan and Matias, 2013).

Although Brazil presents one of the world's largest vegetable biodiversity, (Zeni et al., 2017) at the same time it faces challenges and difficulties in public health. Several studies have been conducted to discover information about local folk medicine, as well as the search for plants that have effective therapeutic activity that may be complementary to modern medicine (Bruning et al., 2012).

There is a growth in the use of herbal remedies for the brazilian population. Two factors could explain this increase. The first would be the advances occurring in the scientific area, which enabled the development of herbal remedies known to be safe and effective. The second is the increasing tendency to search for the population, by less aggressive therapies for primary health care (Yunes et al., 2001).

Thereby, the managers have stimulated the inclusion of medicinal plants, plant derivatives and herbal products in the public health system because it is considered a cheap and easy viable resource available to the Brazilian population (Brasil, 2006). However, more important than stimulating use, preserving this therapeutic knowledge is essential for ethnobotanical and ethno-pharmacological information to be passed on to future generations (Brasileiro et al., 2008).

Marechal Deodoro is one of the poles of planting and marketing of medicinal plants in Alagoas. However, according to the local prefecture and the municipal Council of Health, health professionals have not yet received training for the practices of herbal medicine, and there are no published data on the actual situation of accessibility with this therapy for public health services users.

Therefore, this research aimed to conduct an ethnodirigid study of medicinal plants used by the users of the family health program in Alagoas, Brazil.

\section{Materials and Methods}

The present research was approved by the Research Ethics Committee of the CESMAC University Center under No. 449,467, in accordance with the guidelines of resolution CNS/MS 466/12. This is a descriptive, observational, cross-sectional study. The collection site was the municipality of Marechal Deodoro, which has public health units with the Family Health Program (PSF) in several communities: Poeira, Barro Vermelho, José Dias, Taperaguá, Estiva, Pedras, Massagueira, Barra Nova, Santa Rita, French, Vila Altina, Rua Nova, Terra da Esperança and Malhadas; that do not yet have complementary herbal medicine practices inserted in the health services available to the local population.

For the sample calculation, the criteria of 5\% confidence limit and $99 \%$ confidence interval were used. The hypothetical frequency of the result factor in the population (p) was $50 \%$ since there are no published data on the use of medicinal plants in the municipality surveyed. Based on a population of 13,419 , users enrolled in the PSF. The sample was calculated in 633 individuals, with a $20 \%$ increase so that any losses did not compromise the representativeness of the sample, a total number of 854 individuals were surveyed. Sampling stratification procedures were performed so that the participating users represented their community of origin.

The research subject was recruited by face-to-face verbal invitation at the PSF units during their care hours, as well as during home visits by researchers and community health agents (ACS).

We compiled secondary data, originally obtained through the application of ethnobotanical techniques such as semi-structured and open interviews (Minayo 1994; Dorigoni et al., 2001). The semi-structured and open interviews applied was blocked in four groups: the 1st containing socioeconomic information (sex, age, profession, income, family constitution, place of birth, level of schooling, type of housing); 2st containing information on use of medicinal plants and herbal medicines); and 3st containing information on desire to participate in workshops on herbal medicine.

The data was tabulated in a spreadsheet and analyzed by descriptive statistics for the calculation of frequencies (Soares et al., 2009).

\section{Results}

The interviews were conducted with 852 residents. It was observed that $90 \%$ of the interviewed patients were female, $9 \%$. Male and $5 \%$ chose not to respond. The variable age ranged in the following proportion: $6,80 \%$ had between $29,46 \%$ over 51 years, $25,82 \%$ from 31 to 40 years, $18-20,18,07 \%$ from 41 to 50 years, $16,07 \%$ from 21 to 30 years, and 3, 78\% not informed. Regarding the level of schooling, the vast majority of users, $51 \%$, attended elementary school, $25 \%$ had high school, $16 \%$ did not, only $4 \%$ had the upper level and $4 \%$ were not informed. For $45,07 \%$ of the interviewees the 
monthly family income reaches from one to two minimum wages, $40,37 \%$ has income lower than one minimum wage, $7,51 \%$ has income higher than two minimum wages and $7,51 \%$ not to the item. The majority of respondents, $65,72 \%$, did not report the type of housing that resided, $33,21 \%$ reported living in a masonry house and $1,05 \%$ in Taipa House. In relation to the interviewees ' professions, the majority are housewife with $42,72 \%$, followed by a maid with $9,5 \%$, retired with $7 \%$, autonomous with $4 \%$, Seafood 2, 81\%, Student 2, 69\%, Craftswoman 2, 46 and others/did not inform $0-2 \%$ each (Table 1).
In case of disease $62 \%$ resort to physician, $9 \%$ did not respond, $8.80 \%$ to medicinal plants, $5.86 \%$ resorted to physician and medicinal plants, $4.57 \%$ to ACS, $3.40 \%$ to pharmacy and 0.1 to $3 \%$ responded that they resort to a healer and medicinal plants, medical and pharmacy, Health post, neighbor, nurse or others. In the medical care we highlight the SUS with $94.48 \%$ of search for users, private care with $3.75 \%$ and health plans with $1.76 \%$. Over the healing power, $89.2 \%$ of the users believe that the plants heal, $9.15 \%$ not and 1.65 did not opinate. In the healing power of Phytotherapics $69.13 \%$ believe, 28.4 not and 2.47 did not inform.

Table 1. Socioeconomic profile of users served at the public health units of Marechal Deodoro / AL / Brazil in 2015.

\begin{tabular}{lccc}
\hline & Gender & Sample & Attendance \\
\hline Women & & 765 & 89.58 \\
Male women \% \% TOTAL \% & & 77 & 9.02 \\
Did not answer & Age & 12 & 1.41 \\
\hline & & Sample & Attendance \\
\hline 18 to 20 & & 58 & 6.79 \\
21 to 30 & & 137 & 1604 \\
31 to 40 & & 220 & 25.76 \\
41 to 50 & & 154 & 18.03 \\
51 & Occupation & 221 & 25.88 \\
Did not answer & & 34 & 3.98 \\
\hline & & Sample & Attendance \\
\hline Housewife & 364 & 42.62 \\
Mussel fisherwoman & 24 & 2.81 \\
Self-employed & & 14 \\
Student & & 23 & 1.64 \\
Retired & & 2.69 \\
Domestic worker & 56 & 6.56 \\
Handicraft & & 81 & 9.48 \\
Other: driver, teacher, hairdresser, salesman, fireman, administrative officer, & 21 & 2.46
\end{tabular}

baker, civil servant, businessman, servant, packer, kitchen aid, bartender, director,

lacemaker, motorcycle courier, typist, social worker, maid, washerwoman, agriultor, seamstress, restaurant owner, unemployed, waiter, cook, general services, fisherman, refrigeration technician, community officer, nursing technician, plumber, teacher assistant, clerk, notary, gardener, stonemason, store clerk, radio, manicure, watchman, cashier, cocadeira and production assistant Did not answer

\begin{tabular}{lccc}
\hline & Level of education & Sample & Attendance \\
\hline Non-literate student & & 136 & 15.93 \\
Primary school & & 438 & 51.29 \\
High school & & 214 & 25.06 \\
College degree & Family Income & 36 & 4.22 \\
Did not answer & & 30 & 3.51 \\
\hline & & Sample & Attendance \\
\hline Minimum wage & & 444 & 40.28 \\
1 to 2 salaries & & 484 & 44.96 \\
Twice the minimum wage & Residence structure & 64 & 7.49 \\
Did not answer & & 62 & 7.26 \\
\hline & & Sample & Attendance \\
\hline Masonry & 283 & 33.14 \\
Taipa & 9 & 1.05 \\
Did not answer & 562 & 65.81 \\
\hline
\end{tabular}


Table 1. Continued...

\begin{tabular}{|c|c|c|}
\hline Lives in residence & Sample & Attendance \\
\hline Rent & 124 & 14.52 \\
\hline Own & 667 & 78.10 \\
\hline Others & 35 & 4.10 \\
\hline Did not answer & 28 & 3.28 \\
\hline Family constitution & Sample & Attendance \\
\hline Adults & 293 & $-34: 31$ \\
\hline Elderly & 12 & 1.41 \\
\hline Adults/elderly & 40 & 4.68 \\
\hline Adults / children & 453 & 53.04 \\
\hline Seniors/children & 7 & 0.82 \\
\hline Adults / children / elderly & 36 & 4.22 \\
\hline Did not answer & 13 & 1.52 \\
\hline State & Sample & Attendance \\
\hline Alagoas & 719 & 84.19 \\
\hline Pernambuco & 16 & 1.87 \\
\hline Sergipe & 2 & 0.23 \\
\hline São paulo & 4 & 0.47 \\
\hline Rio de janeiro & 2 & 0.23 \\
\hline Paraíba & 1 & 0.12 \\
\hline Paraná & 1 & 0.12 \\
\hline Bahia & 1 & 0.12 \\
\hline Did not answer & 108 & 12.65 \\
\hline Municipality & Sample & Attendance \\
\hline Rua Marechal Deodoro & 424 & $49-65$. \\
\hline Maceió & 126 & 14.75 \\
\hline São Miguel Dos Campos & 20 & 2.34 \\
\hline $\begin{array}{l}\text { Other: Junqueiro, Atalaia, Pilar, Anadia, Barra de Santo Antônio, Barra de São } \\
\text { Miguel, Arapiraca, Porto Real do Colégio, Santana do Ipanema, Matriz de } \\
\text { Camaragibe, Satuba e Viçosa, Murici, Pão de Açúcar, Taquarana, Chapel, Rio } \\
\text { Largo, Coqueiro Seco, Messias, Jacuípe, União dos Palmares, Mar Vermelho, Porto } \\
\text { Calvo, Santa Rita, São Luiz do Quitunde, Instância, Boca da Mata, Chã Preta, } \\
\text { Cajueiro, Joaquim Gomes, Branquinha, Roteiro, Coruripe, Penedo, Campo Alegre, } \\
\text { Passo de Camaragibe, São José da Lage, Palmeiras dos Indios, Santa Luzia do } \\
\text { Norte, Piacabuçu, São Paulo, Recife, Aracajú, Rio de Janeiro, São José da Lagoa } \\
\text { Tapada, Salvador, Mocambo, Correntes, Garanhus, São João, Águas Belas }\end{array}$ & 145 & 16.98 \\
\hline Did not answer & 139 & 16.28 \\
\hline
\end{tabular}

As observed in graph $1,87.01 \%$ of the interviewees used medicinal plants frequently, and the most cited plants were: the lemongrass (Melissa officinalis) (16\%), Mint (Mentha x piperita) (16\%), Capim Santo (Cymbopogon citratus) (11\%), Boldo (Peumus boldus Molina) (10\%), Aroeira (Schinus Terebinthifolius) (5\%), chamomile (Matricaria chamomilla) (3\%), sweet herb (Pimpinella anisum) (3\%), Barbatimão (Stryphnodendron adstringens) (3\%), Mastruz com (Chenopodium ambrosioides) (3\%) and Eucalyptus (Eucalyptus globulus) (3\%).

It has been found that SUS users mainly use medicinal plants as: calming (16.05\%), digestive (10.74\%), flu $(10.06 \%)$, Antiinflammatory $(7.85 \%)$ and analgesic $(5.80 \%)$. The parts of the plants most commonly used by the user are sheet $(71.24 \%)$, root $(3.46 \%)$ and shell (3.35\%) (Tables 2 and 3).

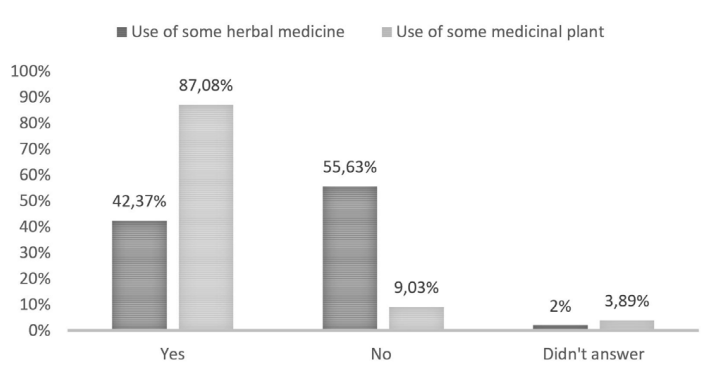

Graph 1. Use of medicinal plants and phytotherapics by users of health units, Marechal Deodoro-AL, Brazil, 2015.

Users gets the plants at home (41.49\%), marketing (16.42\%), with neighbors (12.63\%), in the street $(9.82 \%)$, fair $(2.76 \%)$ and with the family $(1.75 \%) .15 .13 \%$ of users interviewed have not responded to this question. 
Table 2. Relationship of plant medicines used by users of the health units of Marechal Deodoro/AL/Brazil in 2015 with therapeutic use coincides with the scientific literature.

\begin{tabular}{|c|c|c|c|c|c|c|}
\hline \multirow{2}{*}{$\begin{array}{l}\text { Popular Name } \\
\text { - Scientific } \\
\text { Name (s) }\end{array}$} & \multicolumn{3}{|c|}{ Data collected in the community } & \multicolumn{3}{|c|}{ Data from the scientific literature } \\
\hline & Analyzed & Usage: & Popular use & $\begin{array}{l}\text { Part and mode of } \\
\text { use }\end{array}$ & $\begin{array}{l}\text { Indications in } \\
\text { literature }\end{array}$ & REFERENCE \\
\hline $\begin{array}{c}\text { Avocado - } \\
\text { Persea folium }\end{array}$ & Leaf & Tea & $\begin{array}{l}\text { Abdominal } \\
\text { pain, } \\
\text { inflammation, } \\
\text { kidney. }\end{array}$ & $\begin{array}{l}\text { Infusion }-4 g \text { of } \\
\text { fresh leaves in } \\
1 / 2 \text { Cup of water }\end{array}$ & $\begin{array}{c}\text { Diuretic } \\
\text { antidiarrheal }\end{array}$ & $\begin{array}{c}\text { (Cabral et al., } \\
\text { 2014; Lima, } \\
\text { 2008) }\end{array}$ \\
\hline $\begin{array}{l}\text { Watercress - } \\
\text { Nasturtium } \\
\text { officinale }\end{array}$ & Leaf & Tea, Syrup & Flu & $\begin{array}{l}\text { Infusion - } 2 \mathrm{~g} \text { of } \\
\text { dried herb to every } \\
\text { cup of water }\end{array}$ & $\begin{array}{l}\text { Antiseptic, } \\
\text { antimicrobial, } \\
\text { antiscorbutic, } \\
\text { bile duct, lung } \\
\text { condition (cough, } \\
\text { bronchitis) } \\
\text { expectorant. }\end{array}$ & $\begin{array}{l}\text { (Lima, 2008; } \\
\text { Cavalcante, } \\
\text { 2013) }\end{array}$ \\
\hline $\begin{array}{c}\text { Aniseed } \\
\text { and fennel. } \\
\text { Pimpinella } \\
\text { anisum }\end{array}$ & $\begin{array}{l}\text { Leaf and } \\
\text { Flower }\end{array}$ & Tea & $\begin{array}{l}\text { Stomach } \\
\text { Ache }\end{array}$ & $\begin{array}{c}\text { Infusion - } 1.5 \mathrm{~g} \text { dried } \\
\text { fruit in } 150 \mathrm{~mL} \\
\text { water } \\
\text { or } \\
3 \mathrm{~g} \text { of dried seed } \\
\text { (1 Dessert spoon for } \\
\text { each cup of water) in } \\
\text { decoction. }\end{array}$ & $\begin{array}{l}\text { Antidyspeptic and } \\
\text { antispasmodic. }\end{array}$ & $\begin{array}{l}\text { (Lima, 2008; } \\
\text { Brasil, 2010; } \\
\text { Cavalcante, } \\
\text { 2013) }\end{array}$ \\
\hline $\begin{array}{l}\text { Aroeira } \\
\text { - Schinus } \\
\text { terebinthifolius }\end{array}$ & $\begin{array}{l}\text { Leaf and } \\
\text { Flower }\end{array}$ & Tea & $\begin{array}{l}\text { Inflammation } \\
\text { and } \\
\text { cicatrization }\end{array}$ & $\begin{array}{l}\text { Tincture - } 10 \text { to } \\
20 \mathrm{~mL} \text { of tincture } \\
\text { diluted in water. } \\
\text { Decoction - } 2 \mathrm{~g} \\
\text { leaves, one } \\
\text { tablespoon of dried } \\
\text { peel, for internal, } \\
\text { external use and } \\
\text { compresses. }\end{array}$ & $\begin{array}{c}\text { Anti- } \\
\text { inflammatory, } \\
\text { skin conditions, } \\
\text { diuretic. }\end{array}$ & $\begin{array}{c}\text { (Lima, 2008; } \\
\text { Brasil, 2010; } \\
\text { Cavalcante, } \\
\text { 2013) }\end{array}$ \\
\hline $\begin{array}{l}\text { Barbatimão - } \\
\text { Stryphnodendron } \\
\text { barbadetiman }\end{array}$ & $\begin{array}{l}\text { Bark, leaf, } \\
\text { root. }\end{array}$ & Tea & Inflammation & $\begin{array}{c}\text { Tincture - } \\
10 \text { to } 20 \mathrm{ml} \text { of } \\
\text { tincture diluted in } \\
\text { water. } \\
\text { Decoction }-4 \mathrm{~g} \\
\text { of dried peels } \\
\text { ( } 1 \text { tablespoon for } \\
\text { each cup of water). } \\
\text { Dust from the shells } \\
\text { can be used topically } \\
\text { on wounds. } \\
\text { Baths - } 5 \text { mL of } \\
\text { tincture in } 11 \text { of } \\
\text { warm water. }\end{array}$ & $\begin{array}{c}\text { Leucorrhea, } \\
\text { urethral and } \\
\text { vaginal discharge, } \\
\text { hemoptysis } \\
\text { and uterine } \\
\text { bleeding, healing, } \\
\text { astringent, } \\
\text { antidiarrheal, and } \\
\text { scurvy affections }\end{array}$ & $\begin{array}{l}\text { (Lima, 2008; } \\
\text { Brasil, 2010; } \\
\text { Cavalcante, } \\
\text { 2013) }\end{array}$ \\
\hline $\begin{array}{c}\text { Babosa - Aloe } \\
\text { vera, Aloe } \\
\text { barbadensis, } \\
\text { Aloe } \\
\text { perfoliata. }\end{array}$ & Leaf & Tea & $\begin{array}{l}\text { Hair, wound } \\
\text { healing }\end{array}$ & $\begin{array}{l}\text { Juice - the leaf } \\
\text { is cleaned with } \\
\text { alcohol where } \\
\text { some of the green } \\
\text { parts are removed } \\
\text { in order to get to } \\
\text { the gel to brush the } \\
\text { burn/wound. }\end{array}$ & $\begin{array}{c}\text { Healing, } \\
\text { antimicrobial, } \\
\text { laxative. }\end{array}$ & $\begin{array}{c}\text { (Brasil, 2010; } \\
\text { Matos and } \\
\text { Lorenzi, 2002) }\end{array}$ \\
\hline $\begin{array}{l}\text { Boldo from } \\
\text { Chile - Peumus } \\
\text { boldus }\end{array}$ & leaf & Tea & $\begin{array}{l}\text { Gases, poor } \\
\text { digestion, } \\
\text { stomach }\end{array}$ & $\begin{array}{l}\text { Infusion }-1-2 \mathrm{~g} \text { dried } \\
\text { leaf in } 150 \mathrm{~mL} \text { water }\end{array}$ & $\begin{array}{l}\text { Anti-dyspeptic, } \\
\text { cholagogue and, } \\
\text { anti-inflammatory. }\end{array}$ & (Brasil, 2010) \\
\hline
\end{tabular}


Table 2. Continued...

\begin{tabular}{|c|c|c|c|c|c|c|}
\hline \multirow{2}{*}{$\begin{array}{l}\text { Popular Name } \\
\text { - Scientific } \\
\text { Name (s) }\end{array}$} & \multicolumn{3}{|c|}{ Data collected in the community } & \multicolumn{3}{|c|}{ Data from the scientific literature } \\
\hline & Analyzed & Usage: & Popular use & $\begin{array}{l}\text { Part and mode of } \\
\text { use }\end{array}$ & $\begin{array}{c}\text { Indications in } \\
\text { literature }\end{array}$ & REFERENCE \\
\hline $\begin{array}{l}\text { Chamomile - } \\
\text { Chamomilla } \\
\text { recutita, } \\
\text { Matricaria } \\
\text { chamomilla }\end{array}$ & $\begin{array}{l}\text { Flower and } \\
\quad \text { leaf }\end{array}$ & Tea & Anxiolytic & $\begin{array}{c}\text { Internal Use } \\
\text { Infusion - } 3 \mathrm{~g} \text { dried } \\
\text { inflorescences in } \\
150 \mathrm{~mL} \text { water } \\
\text { For external use } \\
\text { Infusion - 6-9g dry } \\
\text { inflorescences } \\
\text { 100ml } \\
\text { Mouthwashes and/or } \\
\text { gargling }\end{array}$ & $\begin{array}{c}\text { Internal use: } \\
\text { antispasmodic, } \\
\text { anxiolytic and } \\
\text { mild sedative } \\
\text { External use: } \\
\text { anti-inflammatory } \\
\text { in disorders of the } \\
\text { oral cavity. }\end{array}$ & (Brasil, 2010) \\
\hline $\begin{array}{l}\text { Laportea- } \\
\text { Urtica diotica }\end{array}$ & Leaf & Tea & Pain & $\begin{array}{c}\text { Infusion }-2 g \\
\text { of dried leaves } \\
\text { ( } 1 \text { tablespoon for } \\
\text { each cup of water) }\end{array}$ & Analgesic & (Lima, 2008) \\
\hline $\begin{array}{l}\text { Holy grass, } \\
\text { lemongrass, } \\
\text { cimic grass, } \\
\text { lemongrass } \\
\text { and lemon } \\
\text { balm - } \\
\text { Cymbopogon } \\
\text { citratus } \|\end{array}$ & Leaf & Tea & $\begin{array}{l}\text { Pain, } \\
\text { sedative, } \\
\text { flu, upset } \\
\text { stomach, } \\
\text { headache. }\end{array}$ & $\begin{array}{l}\text { Infusion - } 1-3 \mathrm{~g} \text { dried } \\
\text { leaf in } 150 \mathrm{ml} \text { water }\end{array}$ & $\begin{array}{l}\text { Antispasmodic, } \\
\text { anxiolytic, } \\
\text { hypotensive, } \\
\text { anticonvulsant, } \\
\text { analgesic, } \\
\text { antihelmintic, } \\
\text { mild anti- } \\
\text { rheumatic } \\
\text { sedative. }\end{array}$ & $\begin{array}{l}\text { (Lima, 2008; } \\
\text { Brasil, 2010; } \\
\text { Cavalcante, } \\
\text { 2013) }\end{array}$ \\
\hline $\begin{array}{l}\text { Lemongrass } \\
\text { - Melissa } \\
\text { officinalis }\end{array}$ & Leaf & Tea & $\begin{array}{l}\text { Sedative, } \\
\text { tummy ache, } \\
\text { pain, cough } \\
\text { and fever. }\end{array}$ & $\begin{array}{l}\text { Infusion- } 1-4 \mathrm{~g} \text { dried } \\
\text { flowering masters in } \\
150 \mathrm{ml} \text { water. } \\
\text { Decoction- } 2 \mathrm{~g} \text { of } \\
\text { leaves and branches } \\
\text { fresh or } 1 \mathrm{~g} \text { of dried } \\
\text { leaves and branches } \\
\text { (1 Dessert spoon for } \\
\text { each cup of water). }\end{array}$ & $\begin{array}{l}\text { Antispasmodic, } \\
\text { anxiolytic and } \\
\text { mild sedative, } \\
\text { digestive, } \\
\text { hyperthyroidism, } \\
\text { topical agent for } \\
\text { cold sores. }\end{array}$ & (Brasil, 2010) \\
\hline $\begin{array}{l}\text { Cologne } \\
\text { - Alpinia } \\
\text { zerumbet }\end{array}$ & Leaf & Tea & $\begin{array}{l}\text { Calming, } \\
\text { heart, high } \\
\text { blood } \\
\text { pressure. }\end{array}$ & $\begin{array}{c}\text {-Dye } 20 \mathrm{~g} \text { dried } \\
\text { leaves in } 100 \mathrm{ml} \\
\text { alcohol } 70 \% \mathrm{p} / \mathrm{p} \\
\text { q.s.p. } \\
\text { Infusion- } 2 \mathrm{~g} \\
\text { (1 Dessert spoon for } \\
\text { each cup of water) } \\
\text { of rhizomes in } \\
\text { decoction. }\end{array}$ & $\begin{array}{l}\text { Analgesic, anti- } \\
\text { inflammatory, } \\
\text { diuretic, } \\
\text { antispasmodic, } \\
\text { uterine stimulant, } \\
\text { antidepressant and } \\
\text { antihypertensive } \\
\text { and } \\
\text { cardioprotective } \\
\text { in cases of mild } \\
\text { hypertension. }\end{array}$ & $\begin{array}{l}\text { (Correa et al., } \\
\text { 2010) }\end{array}$ \\
\hline $\begin{array}{l}\text { Eucalyptus } \\
\text { - Eucalyptus } \\
\text { globules }\end{array}$ & Leaf & Tea & $\begin{array}{l}\text { Fever, flu and } \\
\text { pain. }\end{array}$ & $\begin{array}{l}\text { Infusion- } 1 \mathrm{~g} \text { of dried } \\
\text { herb or } 2 \mathrm{~g} \text { fresh } \\
\text { herb ( } 1 \text { Dessert } \\
\text { spoon for each cup } \\
\text { of water) }\end{array}$ & $\begin{array}{l}\text { Influenza, } \\
\text { bronchitis, } \\
\text { asthma, } \\
\text { expectorant, } \\
\text { rheumatic } \\
\text { diseases, insect } \\
\text { repellent and } \\
\text { antiseptic. }\end{array}$ & (Lima, 2008) \\
\hline
\end{tabular}


Table 2. Continued...

\begin{tabular}{|c|c|c|c|c|c|c|}
\hline \multirow{2}{*}{$\begin{array}{c}\text { Popular Name } \\
\text { - Scientific } \\
\text { Name (s) }\end{array}$} & \multicolumn{3}{|c|}{ Data collected in the community } & \multicolumn{3}{|c|}{ Data from the scientific literature } \\
\hline & Analyzed & Usage: & Popular use & $\begin{array}{c}\text { Part and mode of } \\
\text { use }\end{array}$ & $\begin{array}{l}\text { Indications in } \\
\text { literature }\end{array}$ & REFERENCE \\
\hline $\begin{array}{l}\text { Bristly Starbur- } \\
\text { Acanthospermum } \\
\text { hispidum }\end{array}$ & Leaf, root & Tea & Flu and fever & $\begin{array}{l}\text { Decoction- } 2 \mathrm{~g} \text { of } \\
\text { roots and leaves in } \\
\text { ( } 1 \text { tablespoon for } \\
\text { each cup of water) } \\
\text { Infusion - } 4 \mathrm{~g} \text { of } \\
\text { roots and } \\
\text { Roots leaves in } \\
\text { ( } 1 \text { tablespoon for } \\
\text { each cup of water) } \\
\text { Root - Syrup }\end{array}$ & $\begin{array}{c}\text { Leaf: Anti- } \\
\text { dysmenorrheal, } \\
\text { anti-gonorrhea, } \\
\text { anti dysenteric, } \\
\text { combats } \\
\text { hematuria, } \\
\text { antifebrile } \\
\text { Roots: Dysentery }\end{array}$ & (Lima, 2008) \\
\hline $\begin{array}{l}\text { Ginger - } \\
\text { Zingiber } \\
\text { officinale }\end{array}$ & Roots & Tea & $\begin{array}{c}\text { Flu and sore } \\
\text { throat }\end{array}$ & $\begin{array}{l}\text { Infusion }-0.5-1 \mathrm{~g} \\
\text { dried rhizomes in } \\
150 \mathrm{~mL} \text { water }\end{array}$ & $\begin{array}{l}\text { Anthelmintic, } \\
\text { antacid, } \\
\text { expectorant and } \\
\text { in cases of motion } \\
\text { sickness. }\end{array}$ & (Brasil, 2010) \\
\hline $\begin{array}{l}\text { Common } \\
\text { Guava - } \\
\text { Psidium } \\
\text { guayava }\end{array}$ & Leaf & Tea & $\begin{array}{l}\text { Stomach } \\
\text { Ache }\end{array}$ & $\begin{array}{l}\text { Infusion - } 2 \mathrm{~g} \text { of } \\
\text { dried leaves or } 4 \mathrm{~g} \\
\text { of fresh leaves in } \\
\text { ( } 1 \text { tablespoon for } \\
\text { each cup of water) }\end{array}$ & $\begin{array}{l}\text { Diarrhea, } \\
\text { bactericidal, } \\
\text { fungicidal, } \\
\text { amebicida, } \\
\text { analgesic. }\end{array}$ & (Lima, 2008) \\
\hline $\begin{array}{l}\text { Mint- Mentha } \\
x \text { piperita }\end{array}$ & Leaf & Tea & $\begin{array}{c}\text { Colic, } \\
\text { stomach } \\
\text { ache, flu and } \\
\text { pain }\end{array}$ & $\begin{array}{c}\text { Infusion - } 1.5 \mathrm{~g} \text { dried } \\
\text { leaves and flowering } \\
\text { solids in } 150 \mathrm{~mL} \\
\text { Water }\end{array}$ & $\begin{array}{l}\text { Antispasmodic, } \\
\text { analgesic, irritable } \\
\text { bowel syndrome } \\
\text { and antiflatulent }\end{array}$ & (Brasil, 2010) \\
\hline $\begin{array}{c}\text { Orange - } \\
\text { Citrus sinensis }\end{array}$ & Leaf & Tea & Flu and pain & $\begin{array}{c}\text { Infusion - } \\
2 \text { teaspoons of } \\
\text { leaves for } 1 \text { cup } \\
\text { of tea }\end{array}$ & $\begin{array}{l}\text { Anthelmintic, } \\
\text { anti-hemorrhagic, } \\
\text { anesthetic, } \\
\text { aperient, sedative, } \\
\text { digestive, } \\
\text { depurative, } \\
\text { diuretic, } \\
\text { emmenagogue, } \\
\text { laxative, intestinal } \\
\text { regulator. }\end{array}$ & (Brasil, 2010) \\
\hline $\begin{array}{c}\text { Lemon - Citrus } \\
\times \text { lemon }\end{array}$ & Fruit & Juice & Flu & $\begin{array}{l}\text { Infusion from fruit } \\
\text { or juice. }\end{array}$ & Flu & (Keffuri, 2011) \\
\hline $\begin{array}{c}\text { Bayleaf - } \\
\text { Laurus nobilis }\end{array}$ & Leaf & Tea & $\begin{array}{l}\text { Fever and } \\
\text { intestinal } \\
\text { problems }\end{array}$ & $\begin{array}{l}\text { Infusion - } 4 \mathrm{~g} \text { of } \\
\text { fresh leaves or } 2 \mathrm{~g} \\
\text { of dried leaves } \\
\text { ( } 1 \text { tablespoon for } \\
\text { each cup of water) }\end{array}$ & $\begin{array}{l}\text { Antiseptic } \\
\text { Digestive }\end{array}$ & (Lima, 2008) \\
\hline $\begin{array}{l}\text { Passion fruit } \\
\text {-Passiflora } \\
\text { alata }\end{array}$ & Leaf & Tea & $\begin{array}{l}\text { High Blood } \\
\text { Pressure }\end{array}$ & $\begin{array}{l}\text { Infusion - } 2 \mathrm{~g} \\
\text { of dried leaves } \\
\text { (1 tablespoon for } \\
\text { each cup of water) }\end{array}$ & $\begin{array}{l}\text { Antihypertensive, } \\
\text { anxiolytic. }\end{array}$ & (Lima, 2008) \\
\hline $\begin{array}{l}\text { Wormseed } \\
\text { - Dysphania } \\
\text { ambrosioides }\end{array}$ & Leaf & Tea & $\begin{array}{l}\text { Pain, flu and } \\
\text { intestinal } \\
\text { worms }\end{array}$ & $\begin{array}{l}\text { Tincture }-15 \mathrm{~mL} \\
\text { diluted in water. } \\
\text { Infusion }-4 \mathrm{~g} \\
\text { of fresh plants } \\
\text { (1 tablespoon for } \\
\text { each cup of water) }\end{array}$ & $\begin{array}{l}\text { Muscle pain, } \\
\text { rheumatism, } \\
\text { bronchitis, } \\
\text { bruising, } \\
\text { respiratory } \\
\text { infection, } \\
\text { digestive, } \\
\text { rheumatic pains } \\
\text { and Helminthiasis. }\end{array}$ & (Lima, 2008) \\
\hline
\end{tabular}


Table 2. Continued...

\begin{tabular}{|c|c|c|c|c|c|c|}
\hline \multirow{2}{*}{$\begin{array}{c}\text { Popular Name } \\
\text { - Scientific } \\
\text { Name (s) }\end{array}$} & \multicolumn{3}{|c|}{ Data collected in the community } & \multicolumn{3}{|c|}{ Data from the scientific literature } \\
\hline & Analyzed & Usage: & Popular use & $\begin{array}{c}\text { Part and mode of } \\
\text { use }\end{array}$ & $\begin{array}{c}\text { Indications in } \\
\text { literature }\end{array}$ & REFERENCE \\
\hline $\begin{array}{l}\text { Pitanga } \\
\text { (Brazilian } \\
\text { Cherry) - } \\
\text { Eugenia } \\
\text { uniflora }\end{array}$ & Leaf & Tea & $\begin{array}{l}\text { Stomach } \\
\text { ache }\end{array}$ & $\begin{array}{l}\text { Infusion - } 4 \mathrm{~g} \text { of } \\
\text { fresh leaves or } 2 \mathrm{~g} \\
\text { of dried leaves } \\
\text { ( } 1 \text { tablespoon for } \\
\text { each cup of water) }\end{array}$ & $\begin{array}{c}\text { Fevers, stomach } \\
\text { disorders, } \\
\text { hypertension, } \\
\text { rheumatism, } \\
\text { bronchitis, } \\
\text { sedative, } \\
\text { anti-inflammatory. }\end{array}$ & (Lima, 2008) \\
\hline $\begin{array}{c}\text { Stonebreaker } \\
\text { - Phyllanthus } \\
\text { niruri }\end{array}$ & Leaf & Tea & Kidneys & $\begin{array}{l}\text { Infusion - } 3 \mathrm{~g} \text { of } \\
\text { aerial parts in } \\
150 \mathrm{~mL} \text { water. }\end{array}$ & $\begin{array}{c}\text { Litolytic in } \\
\text { cases of urinary } \\
\text { lithiasis. }\end{array}$ & (Brasil, 2010) \\
\hline $\begin{array}{l}\text { Pomegranate } \\
\text {-Punica } \\
\text { granatum }\end{array}$ & Bark & Tea & Throat & $\begin{array}{l}\text { Infusion - } 5 \mathrm{~g} \text { dried } \\
\text { bark or } 10 \mathrm{~g} \text { fresh } \\
\text { leaves ( } 1 \text { tablespoon } \\
\text { for each cup of } \\
\text { water) }\end{array}$ & $\begin{array}{l}\text { Pharynx and } \\
\text { throat disorders, } \\
\text { heart disease, } \\
\text { diarrhea and } \\
\text { aging. }\end{array}$ & (Lima, 2008) \\
\hline $\begin{array}{l}\text { Coral Plant } \\
\text { - Jatropha } \\
\text { multifida L }\end{array}$ & Sap & Juice & Bleeding & There isn't any & $\begin{array}{l}\text { Cicatrization aid, } \\
\text { anti-hemorrhagic, } \\
\text { laxative, } \\
\text { antirheumatic and } \\
\text { antihypertensive. }\end{array}$ & $\begin{array}{c}\text { (Buch et al., } \\
\text { 2008) }\end{array}$ \\
\hline
\end{tabular}

Table 3. List of medicinal plants utilized byhealth facilities usersfrom Marechal Deodoro / AL / Brazil in 2015with therapeutic use notcoinciding with that of scientific literature.

\begin{tabular}{|c|c|c|c|c|c|c|}
\hline \multirow{2}{*}{$\begin{array}{c}\text { Popular Name } \\
\text { - Scientific } \\
\text { Name (s) }\end{array}$} & \multicolumn{3}{|c|}{ Data collected in the community } & \multicolumn{3}{|c|}{ Scientific literature data } \\
\hline & Part & Usage & $\begin{array}{c}\text { Popular } \\
\text { usage }\end{array}$ & $\begin{array}{c}\text { Part and } \\
\text { usage }\end{array}$ & $\begin{array}{c}\text { Indications in } \\
\text { Literature }\end{array}$ & $\begin{array}{c}\text { Bibliographic } \\
\text { reference }\end{array}$ \\
\hline $\begin{array}{c}\text { Artichoke } \\
\text { - Cynara } \\
\text { cardunculus L. }\end{array}$ & Leaf & Tea & $\begin{array}{c}\text { Abdominal } \\
\text { pain, cramps }\end{array}$ & $\begin{array}{c}\text { Infusion - } 2 \mathrm{~g} \\
\text { fresh leaves or } \\
1 \mathrm{~g} \text { dry leaves } \\
\text { (1 dessert } \\
\text { spoon for } \\
\text { each cup of } \\
\text { water). }\end{array}$ & $\begin{array}{l}\text { Hepatobiliary } \\
\text { disorders, diabetes, } \\
\text { kidney disorders, } \\
\text { antihypertensive }\end{array}$ & (Brasil, 2010) \\
\hline $\begin{array}{l}\text { Rosemary - } \\
\text { Rosmarinus } \\
\text { Officinalis }\end{array}$ & Leaf & Tea & $\begin{array}{c}\text { Poor } \\
\text { digestion, flu }\end{array}$ & $\begin{array}{l}\text { Infusion - } \\
2-3 \mathrm{~g} \text { dry } \\
\text { leaves in } \\
150 \mathrm{~mL} \text { water }\end{array}$ & $\begin{array}{l}\text { Anti-inflammatory and } \\
\text { antiseptic of the oral } \\
\text { cavity. }\end{array}$ & (Brasil, 2010) \\
\hline $\begin{array}{l}\text { African basil } \\
\text { - Ocimum } \\
\text { gratissimum L. }\end{array}$ & Leaf & $\begin{array}{l}\text { Tea, } \\
\text { syrup }\end{array}$ & $\begin{array}{l}\text { Pain, fever, } \\
\text { high blood } \\
\text { pressure and } \\
\text { flu }\end{array}$ & $*$ & Antiseptic, antifungal & (Brasil, 2010) \\
\hline $\begin{array}{l}\text { Blackberry - } \\
\text { Morus nigra, } \\
\text { Morus alba }\end{array}$ & Leaf & Tea & $\begin{array}{c}\text { Hormonal } \\
\text { replacement }\end{array}$ & $\begin{array}{l}\text { Tincture- } \\
10 \text { - } 20 \mathrm{~mL} \text { of } \\
\text { the tincture } \\
\text { diluted in } \\
\text { water } \\
\text { Infusion - } \\
2 \mathrm{~g} \text { of dried } \\
\text { leaves } \\
\text { (1 tablespoon } \\
\text { for each cup } \\
\text { of water) }\end{array}$ & $\begin{array}{l}\text { Disorders of the } \\
\text { mouth, throat and lung, } \\
\text { teeth, skin conditions, } \\
\text { menopausal hot flashes }\end{array}$ & (Lima, 2008) \\
\hline Ampicillin & Leaf & Tea & Inflammation & $*$ & $*$ & $*$ \\
\hline
\end{tabular}

* Data not found in Literature. 
Table 3. Continued...

\begin{tabular}{|c|c|c|c|c|c|c|}
\hline \multirow{2}{*}{$\begin{array}{c}\text { Popular Name } \\
\text { - Scientific } \\
\text { Name (s) }\end{array}$} & \multicolumn{3}{|c|}{ Data collected in the community } & \multicolumn{3}{|c|}{ Scientific literature data } \\
\hline & Part & Usage & $\begin{array}{l}\text { Popular } \\
\text { usage }\end{array}$ & $\begin{array}{c}\text { Part and } \\
\text { usage }\end{array}$ & $\begin{array}{c}\text { Indications in } \\
\text { Literature } \\
\end{array}$ & $\begin{array}{c}\text { Bibliographic } \\
\text { reference }\end{array}$ \\
\hline $\begin{array}{l}\text { Freshcut- } \\
\text { Justicia } \\
\text { pectoralis }\end{array}$ & Tea & Leaf & Headaches & $\begin{array}{l}\text { Juice - the leaf } \\
\text { is sanitized } \\
\text { with alcohol } \\
\text { where part of } \\
\text { the green is } \\
\text { removed so } \\
\text { as to leave } \\
\text { the gel to } \\
\text { brush the } \\
\text { burn / wound. }\end{array}$ & $\begin{array}{c}\text { Cicatrization aid, } \\
\text { antimicrobial, laxative. }\end{array}$ & $\begin{array}{l}\text { (Lima, 2008; } \\
\text { Brasil, 2010; } \\
\text { Cavalcante, } \\
\text { 2013) }\end{array}$ \\
\hline $\begin{array}{l}\text { Common } \\
\text { rue-Ruta } \\
\text { graveolens }\end{array}$ & Leaf & Tea & Inflammation & $\begin{array}{c}\text { Not } \\
\text { recommended } \\
\text { due to } \\
\text { its great } \\
\text { therapeutic } \\
\text { potential }\end{array}$ & $\begin{array}{l}\text { Not recommended due } \\
\text { to its great therapeutic } \\
\text { potential }\end{array}$ & (Lima, 2008) \\
\hline $\begin{array}{l}\text { Indian Shot } \\
\text { Canna Indica }\end{array}$ & Leaf & Tea & Kidneys & $*$ & $*$ & $*$ \\
\hline $\begin{array}{l}\text { Cinnamon - } \\
\text { Cinnamomum } \\
\text { verum }\end{array}$ & Roots & Tea & Anxiolytic & $\begin{array}{l}\text { Infusion: } 1 \mathrm{~g} \\
\text { of dried peels } \\
\text { in } 150 \mathrm{~mL} \\
\text { water }\end{array}$ & $\begin{array}{l}\text { Appetite stimulant, anti- } \\
\text { dyspeptic, antiflatulent } \\
\text { and spasmolytic. }\end{array}$ & (Brasil, 2010) \\
\hline $\begin{array}{l}\text { Black tea } \\
\text {-Camellia } \\
\text { Sinensis }\end{array}$ & Leaf & Tea & $\begin{array}{l}\text { Stomach ache } \\
\text { and poor } \\
\text { digestion }\end{array}$ & $\begin{array}{l}\text { Infusion: } \\
\text { 1gof herb to } \\
100 \mathrm{~mL} \text { of } \\
\text { water }\end{array}$ & $\begin{array}{c}\text { Reduction of } \\
\text { cholesterol levels, } \\
\text { immunostimulatory, } \\
\text { antimicrobial and } \\
\text { antioxidant, prevention } \\
\text { of chronic-degenerative } \\
\text { diseases. }\end{array}$ & $\begin{array}{l}\text { (Nishiyama et al., } \\
\text { 2010) }\end{array}$ \\
\hline $\begin{array}{c}\text { Espinheira- } \\
\text { santa - } \\
\text { Maytenus } \\
\text { ilicifolia }\end{array}$ & Leaf & Tea & Liver & $\begin{array}{l}\text { Infusion }-3 g \\
\text { dried leaves in } \\
150 \mathrm{~mL} \text { water }\end{array}$ & $\begin{array}{l}\text { Antidyspeptic, antacid } \\
\text { and gastric mucosa } \\
\text { protector. }\end{array}$ & (Brasil, 2010) \\
\hline Garrida & Roots & Tea & Infection & $*$ & $*$ & $*$ \\
\hline $\begin{array}{l}\text { Jurubeba- } \\
\text { Solanum } \\
\text { asperolanatum }\end{array}$ & Fruit & Juice & & $\begin{array}{l}\text { Decoction - } \\
\text { Unspecified }\end{array}$ & $\begin{array}{l}\text { Stomach aches, } \\
\text { antiparasitic. }\end{array}$ & $\begin{array}{l}\text { (Di Stasi et al., } \\
\text { 2002) }\end{array}$ \\
\hline $\begin{array}{l}\text { Indian mulberry } \\
\text { Morinda } \\
\text { citrifolia }\end{array}$ & Fruit & Juice & Every part & $*$ & $\begin{array}{c}\text { Anti-bacterial } \\
\text { fruits, analgesic, } \\
\text { anti-congestitive, } \\
\text { antioxidant, } \\
\text { expectorant, } \\
\text { anti-inflammatory, } \\
\text { astringent, emollient, } \\
\text { emmenagogue, } \\
\text { laxative, hypotensive, } \\
\text { blood purifier, } \\
\text { immunostimulant and } \\
\text { tonic. Anti-cancer } \\
\text { action is also attributed } \\
\text { to the fruit }\end{array}$ & (Brasil, 2010) \\
\hline
\end{tabular}

* Data not found in Literature. 
Table 3. Continued...

\begin{tabular}{|c|c|c|c|c|c|c|}
\hline \multirow{2}{*}{$\begin{array}{c}\text { Popular Name } \\
\text { - Scientific } \\
\text { Name (s) }\end{array}$} & \multicolumn{3}{|c|}{ Data collected in the community } & \multicolumn{3}{|c|}{ Scientific literature data } \\
\hline & Part & Usage & $\begin{array}{l}\text { Popular } \\
\text { usage }\end{array}$ & $\begin{array}{c}\text { Part and } \\
\text { usage }\end{array}$ & $\begin{array}{c}\text { Indications in } \\
\text { Literature }\end{array}$ & $\begin{array}{c}\text { Bibliographic } \\
\text { reference }\end{array}$ \\
\hline $\begin{array}{c}\text { Elderberry - } \\
\text { Sambucus nigra }\end{array}$ & Flower & Tea & $\begin{array}{l}\text { Stomach } \\
\text { aches, flu }\end{array}$ & $\begin{array}{l}\text { Infusion - } \\
3 \mathrm{~g} \text { of dried } \\
\text { flowers in } \\
150 \mathrm{ml} \text { of } \\
\text { water }\end{array}$ & Diaphoretic & (Brasil, 2010) \\
\hline $\begin{array}{c}\text { Comb Hyptis } \\
\text { - Hyptis } \\
\text { pectinata }\end{array}$ & Leaf & Tea & Flu & $*$ & $\begin{array}{l}\text { Antiedematogenic and } \\
\text { contraceptive. }\end{array}$ & $\begin{array}{l}\text { (Melo et al., } \\
\text { 2001) }\end{array}$ \\
\hline Foot of thorn & Leaf & Tea & Flu & $*$ & $*$ & $*$ \\
\hline For everything & Leaf & Tea & Flu & $*$ & $*$ & $*$ \\
\hline
\end{tabular}

* Data not found in Literature.

The transmission of knowledge about the use of these medicinal plants and herbal remedies did mainly within the family environment (82\%). Also was quoted as important in the transmission of this knowledge about the use of medicinal plants the neighbors $(5.63 \%)$ and the doctors, nurses, books, radio, television and ACS, with relative frequency less than $2 \%$.

Of those surveyed, $79.69 \%$ do not usually store the plants before the preparation, $17.28 \%$ store. The others have not responded to this question. Of the total respondents who answered that store the plants before the preparation, only $44.89 \%$ specify the location where the usually store. The most cited were: the cooler (18\%-for medicinal plants and $37 \%$ for herbal medicines) and the plastic bag (10\% for medicinal plants).

It was found that only a small portion $(13.26 \%)$ the community makes use of medicinal plant or medicine herbal medicines during treatment with allopathic drugs (Graph 2) such as paracetamol (11.50\%), analgesics (4.42\%) or anti-inflammatory drugs $(3.53 \%)$. The majority of respondents $(64 \%)$ no answer to that question and a range of 1 to $3 \%$ cited the use of antibiotics, birth control, dypirone, omeprazole, anador, sedative, multigripe, diclofenac, diazepam, anador, hidromed, company and dipyrone.

It is noteworthy that only $1.52 \%$ of respondents claimed to have presented some type of allergy to medicinal plants or herbal medicines. The plants cited in this case were: lemon grass $(7.69 \%)$, nettle (7.69\%), Aroeira $(7.69 \%) .69 .24 \%$ of respondents who claimed to have had this effect, did not inform the responsible medicinal plant. No phytotherapic medication was reported by these volunteers.

It was observed that $56.80 \%$ and $38.14 \%$ of the users are satisfied with the results obtained in the treatment with medicinal plants or herbal medicines, respectively (Graph 3).

It was also found that $85.56 \%$ of the interviewees never received any informative material, such as a folder, booklet or pamphlets, on phytotherapy. Only $12.32 \%$ claimed to have received this type of material and $2.12 \%$ did not answer that question. Lastly, it was questioned whether the interviewees would like to participate in workshops and/or lectures on plants with medicinal properties or

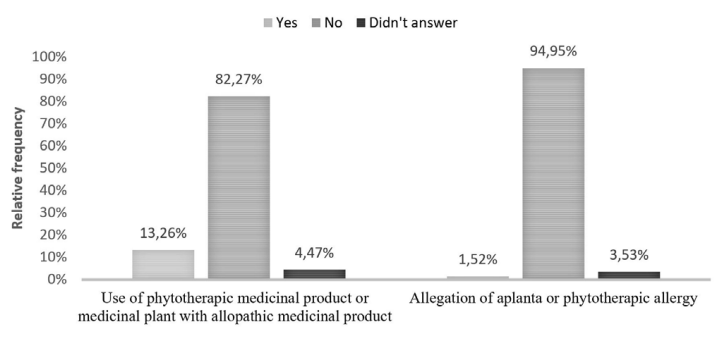

Graph 2. Use of phytotherapic medicine or medicinal plant with allopathic medication by users of health units, Marechal Deodoro-AL, Brazil, 2015.

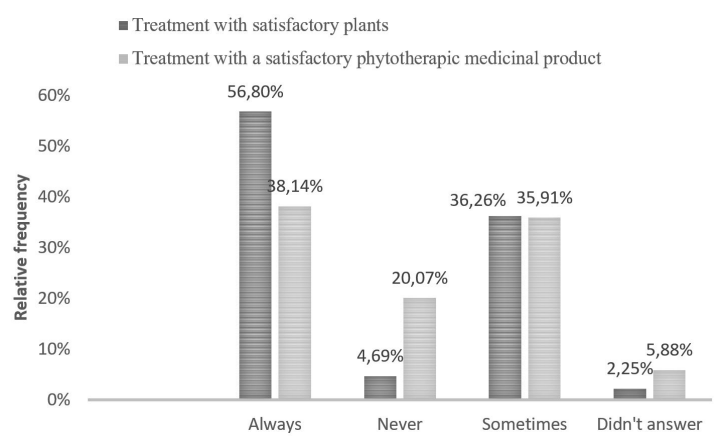

Graph 3. Satisfaction index related to medicinal plants and medicinal plants by the users of health units, Marechal Deodoro-AL, Brazil, 2015.

herbal medicines, and $62.2 \%$ said they had an interest in participating. $35.56 \%$ said they would not want to participate and $2.24 \%$ did not.

\section{Discussion}

The use of medicinal plants is increasingly being the target of scientific research, which in most cases aims to detail this popular culture in a community, ranging from knowledge about the use of plants and herbal medicines used, to evidence of its therapeutic and prophylactic potential, and to the way this knowledge propagates through generations. 
In Marechal Deodoro, little instruction to the users was given by healthcare professionals, causing the exclusion of said instruction as an offer to users of public healthcare services. This situation was confirmed by our research, although the majority $(86.89 \%)$ of the users utilized medicinal plants to treat their diseases, they did so mainly through family encouragement $(82.32 \%)$ and not through professional.

The results obtained in this research indicated that women continue to be the holders of this folk knowledge which corroborate with results from previous research, where the highest number interviewees are females. Another possible explanation for this is that men were absent from home or the healthcare units for work reasons (Melo et al. 2001; Arnous et al. 2005; Marinho et al., 2011). The interviewed women were mostly housewives, which explains their in-person availability to participate in the research. Similar results were observed in other municipalities in Brazil, such as Sobral, where $66.7 \%$ of interviewees were women and housewives (Nascimento et al., 2013).

It can be affirmed that the majority of users who benefited from therapeutic manipulations based on the use of medicinal plants had elementary education, a finding observed in other cities such as Governador Valadares (56.40\%), and Mirassol D'Oeste (55\%) (Brasileiro et al., 2008; Carniello et al., 2010). This population with a low level of knowledge ends up limited to the knowledge passed by family members, and therefore the transmission of correct knowledge to the future generations guarantees the appropriate use.

When a health problem was present, the majority $(61.36 \%)$ of the users from Marechal Deodoro would first resort to the doctor, who mainly prescribed allopathic medicines. Only $17.33 \%$ of the users reported the medicinal plants as the first option and $5.85 \%$ made the associated use of allopathic drugs and complementary therapies without indication, representing a risk to the population. Differing data were observed in the town of Manejo, where the behavior adopted in the face of disease in a family member was not the search for traditional medicine, as in Marechal Deodoro, but medicinal plant preparations indicated by relatives, as the first option being this millennial therapeutical form (Oliveira and Menini Neto, 2012).

Overall, $86.89 \%$ of users reported the use of medicinal plants at some point for the treatment of their diseases. This high utilization rate found in the research corroborated with those obtained in other communities from Minas Gerais (90\%) (Carvalho et al., 2013), Teutônia (92.9\%) (Schwambach and Amador, 2007) and Juvenilia (97\%), where a large part of this population sought alternative measures due to the ease of acquisition of medicinal plants, the influence of the family tradition and the high cost of allopathic medicines. In Brazil, due to the diversity of its biome, it is frequent to observe several types of plants with medicinal properties (Costa and Mayworm, 2011).

Lemon grass (Melissa officinalis L.), mint (Menthax piperita) and holy grass (Cymbopogon citratus) are among the most cited medicinal plants not only by the population of
Marechal Deodoro but also by other Brazilian communities living in Manejo - Lima Duarte (Oliveira and Menini Neto, 2012) and Governador Valadares (Brasileiro et al., 2008).

Lemon grass is a plant traditionally used to induce calmness, reduce stress and improve cognition for it is proven to be a mild sedative and anxiolytic. It works on abdominal cramps (antispasmodic) and symptoms of indigestion (anti-dyspeptic). The recommendations for mint are the same as lemon grass (anti-dyspeptic and antispasmodic). Guaco syrup was indicated in this research as the most used herbal medicine, it is composed of the hydroalcoholic extract of Mikania glomerata, aids in the treatment of respiratory tract disorders such as persistent coughs and coughs with expectoration. It has as its main component coumarin, to which the effect of bronchial dilation and elimination of respiratory secretions through coughing is attributed (Lucena et al., 2015; Lima et al., 2013; Zago et al., 2009; Nunes et al., 2015).

The purposes of using the medicinal plants indicated in this study were similar to those determined in the studies carried out in the community health center (PAM) of the municipal government of Passo Fundo (Silva and Hahn, 2011) and in Cascavel - Paraná (Tomazzoni et al., 2006), where the most frequently mentioned therapeutic properties were digestive $(76 \%)$, sedative $(43 \%)$ and anti-influenza (37\%).

Of the commonly used parts, the leaves received the higher frequency of use citation due to the fact they are exposed in most plants, and can be used at any time of the year. Other structures may present seasonality as is the case of the flower (Botelho et al., 2014), in addition to the fact that the harvesting of leaves does not pose an immediate risk to the plant.

Of the 44 species cited, $61 \%$ had their popular use coinciding with that cited in the literature and two species cited by the popular name, were not found in the literature (pé de espinho and ampicilina). Indian mulberry (Morinda citrifolia) was cited as a "jack of all trades" plant, with no standardization of use and therefore was classified as misused. Coralbush (Jatropha multifida) still has its toxicity and pharmacological action as the target of studies (Buch et al., 2008). Some plants have their therapeutics correctly cited by users, but with divergence of scientific findings in the method of use. As is the case with the freshcut (Justicia pectoralis) (Cavalcante, 2013), jurubeba (Solanum asperolanatum) (Di Stasi et al., 2002). Common rue (Ruta graveolens) does not have its use recommended, due to the strong therapeutic action in the organism (Lima, 2008).

The correct use of medicinal plants was observed in the study carried out in Campina Grande - Paraíba, where the interviewees used medicinal plants and herbal medicines safely, because $21.42 \%$ obtained this knowledge from living with friends, $7.14 \%$ with relatives and $57.13 \%$ with their parents. In addition, $7.14 \%$ of those interviewed learned this folk culture by helping older people prepare and sell the herbs or learned by themselves with the daily practice of sales in the workplace (França et al., 2008). 
It was possible to observe that the users still maintain the habit of cultivating the medicinal plants in their residences, which is certainly consistent with the familial transmission of the use of these vegetable species. Corroborating with this information are several researches showing that the use of medicinal plants is facilitated through their obtention, being cultivated by the users themselves in their backyards or gardens, facilitating immediate consumption where they are probably still used fresh (Botelho et al., 2014; Carvalho et al., 2013; Marinho et al., 2011).

In learning about the use of plants, research confirms the theory that therapeutic use is part of a knowledge and tradition passed on from generation to generation, since knowledge about home-made preparations with medicinal plants has been passed on by some family member, probably the oldest one in the family (Nascimento et al., 2013). Studies such as the one in the municipality of Teutônia in Rio Grande do Sul and in São José de Espinhares in Paraíba describes that learning about the use of medicinal plants was acquired through the parents (Brasil, 2004; Schwambach and Amador, 2007).

In relation to storage it is stated that fresh plants should not remain in the refrigerator for more than a week inside plastic bags and neither the dried ones kept for more than three months (Matos and Lorenzi, 2002). Due to the lack of technical and scientific guidelines on the storage of plants after their preparation and use, and to the variation of the types of preparations, it is recommended to use them right after the preparation and not to reutilize the plant in other manipulations. In this study, it was observed that even without having access to scientific information most users did not usually store the plants before their preparation, which brings benefits to the maintenance of their health. This behavior was also observed in relation to herbal remedies, which still have the advantage of greater stability, due to the chemical processing to which they were submitted.

Incorrect usage of plants or herbal medicines can cause adverse reaction or intoxication and being able to identify them properly is very important. There is an insufficient supply of technical information for most medicinal plants, so as to ensure quality, efficacy and safety of their use, and factors such as the use of fresh plants, temperature, sun exposure, drying, packaging can affect quality and consequently the therapeutic value of these plants (Arnous et al., 2005; Calixto, 2000; Siqueira et al., 2015).

We must be careful with the use of allopathic medicines and herbal remedies since the mixture of both can generate overdosage, inhibitive effect or adverse reaction. The Ministry of Health uses pharmacovigilance of herbal medicine and spontaneous notification of reactions to medications, as well as monitoring patients who use conventional medicines and phytoterapeutics (Capasso et al., 2000; Veiga-Junior and Mello, 2008; Cordeiro et al., 2005; Amorim et al., 2007).

With the lack of scientific basis and knowledge about the correct use and manipulations of plants and herbal medicines we noticed a great difference between the levels of satisfaction among them. What is striking is that by seeing to the need of the majority, medicinal plants and herbal remedies tend to remain active, perpetuating themselves from generation to generation.

Health at the primary level is focused on health promotion where this information is spread through lectures in waiting rooms and group activities, generating interest and curiosity in the population about the proposed theme. In the study it was noticed that $62.06 \%$ of those who attend the units are interested in the subject. However, there is a shortage of educational actions aimed at the use of medicinal plants and herbal remedies on behalf of the managers, portraying the profile of a population whose health care culture, inherited from a family history, is memorable and that they believe that their self-knowledge is sufficient to supply their infirmities by making empirical use of these medications (Nascimento et al., 2013).

Despite being satisfied with the use of medicinal plants, there is a lack of stimulation for the safe usage of herbal medicine being necessary to rescue and exploit this knowledge not only for the population, but for professionals who work directly with it, since there is a need for actions that involve this type of alternative practice, since it is inserted and well diffused in the sociocultural context of these subjects.

\section{Conclusion}

In the community searched is observed to tradition of its inhabitants to get by natural resources for curing diseases. The large number of plants cited in this work (44) reaffirms the importance of ethnobotany research in the rescue traditional knowledge; either by the cultural historical value is due to the need to confirm the indications of use. The main way of preparing medicines is through teas using leaves and/or part area of plants. This work is expected to contribute with a proposal for guidance on the use of medicinal plants in the system public health.

\section{References}

AMORIM, M.F.D., DINIZ, M.F.F.M., ARAÚJO, M.S.T., PITA, J.C.L.R., DANTAS, J.G., RAMALHO, J.A., XAVIER, A.L., PALOMARO, T.V. and JÚNIOR, N.L.B., 2007. The controvertible role of kava (Piper methysticum G. Foster) an anxiolytic herb, on toxic hepatitis. Revista Brasileira de Farmacognosia, vol. 17, pp. 448-454. http://dx.doi.org/10.1590/S0102-695X2007000300020.

ARNOUS, A.H., SANTOS, A.S., PASSOS, R. and BEINNER, C., 2005. Plantas medicinais de uso caseiro-conhecimento popular e interesse por cultivo comunitário medicinal. Espaço Saúde, vol. 62, pp. 1-6.

BOTELHO, J., LAMANO-FERREIRA, A.P.N. and FERREIRA, M.L., 2014. Prática de cultivo e uso de plantas domésticas em diferentes cidades brasileiras. Ciência Rural, vol. 44, no. 10, pp. 1810-1815. http://dx.doi.org/10.1590/0103-8478cr20131036.

BRAGANÇA, A.L.R., 1996. Plantas medicinais antidiabéticas: uma abordagem multidisciplinar. Niterói: EDUFF.

BRASIL. Ministério da Saúde, 2004. HumanizaSUS: Politica Nacional de Humanização: documento base para gestores do 
SUS. 2. ed. Brasília: Ministério da Saúde, 49 p. Série B. Textos Básicos de Saúde.

BRASIL. Ministério da Saúde, 2006. Portaria $n^{\circ}$ 971, de 3 de maio de 2006. Aprova a Politica Nacional de Práticas Integrativas e Complementares no Sistema Único de Saúde. Diário Oficial da República Federativa do Brasil, Brasília, 4 maio. Seção 1, p. 19.

BRASIL. Agência Nacional da Vigilância Sanitária, 2010 [viewed 20 December 2018]. Farmacopéia Brasileira [online]. 5. ed. Brasília: Agência Nacional de Vigilância Sanitária. Available from: http://portal.anvisa.gov.br/documents/33832/260079/5\% C2\%AA+edi $\%$ C3\%A7\%C3\%A3o+-+Volume+1/4c530f86-fe $83-$ $4 \mathrm{c} 4 \mathrm{a}-\mathrm{b} 907-6 \mathrm{a} 96 \mathrm{~b} 5 \mathrm{c} 2 \mathrm{~d} 2 \mathrm{fc}$

BRASILEIRO, B.G., PIZZIOLO, V.R., MATOS, D.S., GERMANO, A.M. and JAMAL, C.M., 2008. Plantas medicinais utilizadas pela população atendida no 'Programa de Saúde da Família', Governador Valadares, MG, Brasil. Revista Brasileira de Ciências Farmacêuticas, vol. 44, no. 4, pp. 629-636. http:// dx.doi.org/10.1590/S1516-93322008000400009.

BRUNING, M.C.R., MOSEGUI, G.B.G. and VIANNA, C.M.M., 2012. A utilização da fitoterapia e de plantas medicinais em unidades básicas de saúde nos municípios de Cascavel e Foz do Iguaçu - Paraná: a visão dos profissionais de saúde. Ciencia \& Saude Coletiva, vol. 17, no. 10, pp. 2675-2685. http://dx.doi. org/10.1590/S1413-81232012001000017. PMid:23099755.

BUCH, D.R.,ARANTES, A.B. and CAMPELO, P.M.S., 2008. Verificação da atividade cicatrizante do exudato de folhas de Jatropha multifida L. Revista Brasileira de Farmácia, vol. 89, pp. 142-145.

CABRAL, C., PITA, J.R. and SALGUEIRO, L., 2014. Plantas medicinais: entre o passado e o presente: a colecção de fármacos vegetais da Faculdade de Farmácia da Universidade de Coimbra (séculos $X I X-X X$ ). 2. ed. Coimbra: Imprensa da Universidade de Coimbra. http://dx.doi.org/10.14195/978-989-26-0875-4.

CALIXTO, J.B., 2000. Efficacy, safety, quality control, marketing and regulatory guidelines for herbal medicines (phytotherapeutic agents). Brazilian Journal of Medical and Biological Research, vol. 33, no. 2, pp. 179-189. http://dx.doi.org/10.1590/S0100879X2000000200004. PMid:10657057.

CAPASSO, R., IZZO, A.A., PINTO, L., BIFULCO, T., VITOBELLO, C. and MASCOLO, N., 2000. Phytotherapy and quality of herbal medicines. Fitoterapia, vol. 71, suppl. 1, pp. 58-65. http://dx.doi.org/10.1016/S0367-326X(00)00173-8. PMid:10930714.

CARNIELLO, M.A., SILVA, R., CRUZ, M.A.B. and GUARIM NETO, G., 2010. Quintais urbanos de Mirassol D'Oeste-MT, Brasil: uma abordagem etnobotânica. Acta Amazonica, vol. 40, no. 3, pp. 451-470. http://dx.doi.org/10.1590/S004459672010000300005

CARVALHO, J.S.B., MARTINS, J.D.L., MENDONÇA, M.C.S.M. and LIMA, L.D., 2013. Uso popular das plantas medicinais na comunidade da Várzea, Garanhuns-PE. Revista de Biologia e Ciências da Terra, vol. 13, no. 2, pp. 58.

CAVAlCANTE, R., 2013. Fitodontologia. 1. ed. Rio Branco: Editora do autor.

COAN, C.M. and MATIAS, T., 2013. A utilização das plantas medicinais pela comunidade indígena de Ventarra Alta- RS. Revista de Educação do IDEAU., vol. 8, no. 18, pp. 1-13.

CORDEIRO, C.H.G., CHUNG, M.C. and SACRAMENTO, L.V.S., 2005. Interações medicamentosas de fi toterápicos e fármacos: Hypericum perforatum e Piper methysticum. Revista Brasileira de Farmacognosia, vol. 15, no. 3, pp. 272-278. http:// dx.doi.org/10.1590/S0102-695X2005000300019.

CORREA, A.J.C., LIMA, C.E. and COSTA, M.C.C.D., 2010. Alpinia zerumbet (Pers.) B. L. Burtt \& R. M. Sm. (Zingiberaceae): levantamento de publicações nas áreas farmacológica e química para o período de 1987 a 2008. Revista Brasileira de Plantas Medicinais. vol. 12, no. 1, p. 113-119.

COSTA, V.P. and MAYWORM, M.A.S., 2011. Plantas medicinais utilizadas pela comunidade do bairro dos Tenentes - município de Extrema, MG, Brasil. Revista Brasileira de Plantas Medicinais, vol. 13, no. 3, pp. 282-292. http://dx.doi.org/10.1590/S151605722011000300006

DI STASI, L.C., OLIVEIRA, G.P., CARVALHAES, M.A., QUEIROZ-JUNIOR, M., TIEN, O.S., KAKINAMI, S.H. and REIS, M.S., 2002. Medicinal plants popularly used in the Brazilian Tropical Atlantic Forest. Fitoterapia, vol. 73, no. 1, pp. 69-91. http://dx.doi.org/10.1016/S0367-326X(01)00362-8. PMid:11864767.

DORIGONI, P.A., GHEDINI, P.C., FRÓES, L.F., BAPTISTA, K.C., ETHUR, A.B.M., BALDISSEROTTO, B., BURGER, M. E., ALMEIDA, C.E., LOPES, A.M.V. and ZÁCHIA, R.A., 2001. Levantamento de dados sobre plantas medicinais de uso popular no município de São João do Polêsine, RS, Brasil. I - Relação entre enfermidades e espécies utilizadas. Revista Brasileira de Plantas Medicinais, vol. 4, no. 1, pp. 69-79.

ELDIN, S. and DUNFORD, A. 2001. Fitoterapia na atenção primária a saúde. São Paulo: Manole.

FRANÇA, I.S.X., SOUZA, J.A., BAPTISTA, R.S. and BRITTO, V.R.S., 2008. Medicina popular: benefícios e malefícios das plantas medicinais. Revista Brasileira de Enfermagem, vol. 61, no. 2, pp. 201-208. http://dx.doi.org/10.1590/S0034-71672008000200009. PMid: 18572840

JACOBY, C., COLTRO, E.M., SLOMA, D.C., MULLER, J., DIAS, L.A., LUFT, M. and BERUSKI, P., 2002. Plantas medicinais utilizadas pela comunidade rural de Guamirim, Município de Irati, PR. Revista Ciências Exatas e Naturais, vol. 4, no. 1, pp. 79-89.

KEFFURI, C.W., 2011. Caderno das nossas plantas medicinais. 1. ed. Viçosa: Universidade Federal de Viçosa.

LIMA, A., 2008. Índice Terapêutico Fitoterápico: Ervas Medicinais. 1. ed. Petrópolis: EPUB, $328 \mathrm{p}$

LIMA, L.L., et al, 2013. A Prática da fitoterapia a partir do conhecimento popular em três comunidades do Valentina, João Pessoa - Paraíba. Revista Ciência Saúde Nova Esperança, vol. 11 , no. 3, pp. 20-31

LUCENA, B.F.F., et al, 2015. Avaliação da atividade antibacteriana e moduladora de aminoglicosídeos do óleo essencial de Cymbopogon citratus (DC.) Stapf. Acta Biologica Colombiana, vol. 20, no. 1, pp. 39-45.

MARINHO, M.G., SILVA, C. and ANDRADE, L.H., 2011. Levantamento etnobotânico de plantas medicinais em área de caatinga no município de São José de Espinharas, Paraíba, Brasil. Revista Brasileira de Plantas Medicinais, vol. 13, no. 2, pp. 170-182. http://dx.doi.org/10.1590/S1516-05722011000200008.

MATOS, F.J.A. and LORENZI, H., 2002. Plantas Medicinais no Brasil: nativas e exóticas. 1st ed. São Paulo: Instituto Plantarum de Estudos da Flora. 
MELO, G.B., SILVA, R.L., ANTONIOLLI, A.R., MELO, V.A., LIMA, S.O., SILVA, P.M. and SILVA JUNIOR, O.C., 2001. Efeitos do extrato aquoso da Hyptis pectinata sobre a regeneração hepática após hepatectomia parcial de $70 \%$ : resultados preliminares. Acta Cirurgica Brasileira, vol. 16, suppl. 1, pp. 13-15. http:// dx.doi.org/10.1590/S0102-86502001000500005.

MINAYO, M.C.S., 1994. Pesquisa social: teoria método e criatividade. 17. ed. Petrópolis: Vozes, 80 p.

NASCIMENTO, W.M.C., MELO, O.F., SILVA, I.F. and SOUZA, F.L., 2013. Plantas medicinais e sua utilização pelas comunidades do município de Sobral, Ceará. Sanare, vol. 12, no. 1, pp. 46-53.

NISHIYAMA, M.F., COSTA, M.A.F., COSTA, A.M., SOUZA, C.G.M., BÔER, C.G., BRACHT, C.K. and PERALTA, R.M., 2010. Chá verde brasileiro (Camellia sinensis var assamica): efeitos do tempo de infusão, acondicionamento da erva e forma de preparo sobre a eficiência de extração dos bioativos e sobre a estabilidade da bebida. Food Science and Technology, vol. 30, suppl. 1, pp. 191-196. http://dx.doi.org/10.1590/S0101-20612010000500029.

NOLLA, D., SEVERO, B.M.A. and MIGOTT, A.M.B.M., 2005. Plantas Medicinais. 2. ed. Passo Fundo: UPF.

NUNES, M.G.S.; BERNARDINO, A.O. and MARTINS, R.D., 2015. Uso de plantas medicinais por pessoas com hipertensão. Revista Rene, vol. 16, no. 6, pp. 775-781.

OLIVEIRA, E. and MENINI NETO, L., 2012. Levantamento etnobotânico de plantas medicinais utilizadas pelos moradores do povoado de Manejo, Lima Duarte - MG. Revista Brasileira de Plantas Medicinais, vol. 14, no. 2, pp. 311-320. http://dx.doi. org/10.1590/S1516-05722012000200010.

SCHWAMBACH, K.H. and AMADOR, T.A., 2007. Estudo da utilização de plantas medicinais e medicamentos em um Município do Sul do Brasil. The American Journal of Pharmacy, vol. 26, no. 4 , pp. 602-608.

SILVA, B.Q. and HAHN, S.R., 2011. Uso de plantas medicinais por indivíduos com hipertensão arterial sistêmica, diabetes mellitus ou dislipidemias. Revista Brasileira de Farmácia Hospitalar e Serviços de Saúde, vol. 2, pp. 36-40.
SIQUEIRA, B., ESCOBAR, P., LACERDA, G. and DAMASCENO, E., 2015. Estudo etnobotânico de plantas medicinais utilizadas pela população atentida no programa saúde da família no município de Juvenília, Minas Gerais. Revista Brasileira de Pesquisa em Ciências da Saúde, vol. 1, no. 2, pp. 39-45.

SOARES, M.A.A., BRAGA, J.R.P., MOURÃO, A.E.B., PARENTE, K.M.S. and PARENTE FILHO, E.G. 2009. Levantamento etnobotânico das plantas medicinais utilizadas pela população do município de Gurinhém - Paraíba. Revista Homem, Espaço e Tempo, vol. 3, n. 2, pp. 36-47.

TOMAZZONI, M.I., NEGRELLE, R.R.B. and CENTA, M.L., 2006. Fitoterapia popular: a busca instrumental enquanto prática terapêuta. Texto \& Contexto, vol. 15, no. 1, pp. 115-121. http:// dx.doi.org/10.1590/S0104-07072006000100014.

VEIGA-JUNIOR, V.F. and MELLO, J.C.P., 2008. As monografias sobre plantas medicinais. Revista Brasileira de Farmacognosia, vol. 18, no. 3, pp. 464-471. http://dx.doi.org/10.1590/S0102$695 \times 2008000300022$

YUNES, R.A., PEDROSA, R.C. and CECHINEL, F.V., 2001. Fármacos e fitoterápicos: a necessidade do desenvolvimento da indústria de fitoterápicos e fitofármacos no Brasil. Quimica Nova, vol. 24, no. 1, pp. 147-152. http://dx.doi.org/10.1590/ S0100-40422001000100025.

ZAGO, J.A.A., USHIMARU, P.I., BARBOSA, L.N. and FERNANDES JUNIOR, A., 2009. Sigergismo entre óleos essenciais e drogas antimicrobianas sobre linhagens de Staphylococcus aureus e Escherichia coli isoladas de casos clínicos humanos. Revista Brasileira de Farmacognosia, vol. 19, no. 04, pp. 828-833. http:// dx.doi.org/10.1590/S0102-695X2009000600005.

ZENI, A.L.B., PARISOTTO, A.V., MATTOS, G. and HELENA, E.T.S., 2017. Utilização de plantas medicinais como remédio caseiro na Atenção Primária em Blumenau, Santa Catarina, Brasil. Ciencia \& Saude Coletiva, vol. 22, no. 8, pp. 27032712. http://dx.doi.org/10.1590/1413-81232017228.18892015. PMid:28793084. 